\title{
La sensibilité esthétique et le jugement de goût
}

\section{Edouard Aujaleu}

\section{OpenEdition}

\section{Journals}

\section{Édition électronique}

URL : http://journals.openedition.org/trema/1921

DOI : 10.4000/trema.1921

ISSN : 2107-0997

\section{Éditeur}

Faculté d'Éducation de l'université de Montpellier

\section{Édition imprimée}

Date de publication : 1 avril 1997

Pagination : 33-52

ISSN : 1167-315X

\section{Référence électronique}

Edouard Aujaleu, «La sensibilité esthétique et le jugement de goût », Tréma [En ligne], 11 | 1997, mis en ligne le 01 avril 1997, consulté le 19 avril 2019. URL : http://journals.openedition.org/trema/1921 ; DOI : 10.4000/trema. 1921

Ce document a été généré automatiquement le 19 avril 2019.

Trema 


\title{
La sensibilité esthétique et le jugement de goût
}

\author{
Edouard Aujaleu
}

1 En Occident, une longue tradition considère l'appréciation de la beauté comme une affaire intellectuelle. De Platon à Hegel, tout un courant philosophique a voulu voir dans la beauté une manifestation de la vérité. Mais ce faisant on évacue le fait que l'appréciation esthétique renvoie d'abord aux sens, et pas seulement à la vue et à l'ouïe. Le prétendu privilège de ces deux sens n'est qu'un moyen d'accentuer l'aspect intellectuel de l'expérience esthétique. «Beaucoup de philosophies se réfèrent à la vue ; peu à l'ouie ; moins encore donnent leur confiance au tactile, comme à l'odorat. L'abstraction découpe le corps sentant, retranche le goût, l'odorat et le tact, ne garde que la vue et l'ouïe, intuition et entendement. Abstraire signifie moins quitter le corps que le déchirer en morceaux : analyse ${ }^{1}$.

2 Nous voudrions essayer de déterminer ce que peut être une sensibilité esthétique : est-ce une sensibilité spécifique ? Dans quelle mesure permet-elle un jugement de goût ? Celui-ci est-il radicalement subjectif ou comporte-t-il une part d'universalité ? Est-il susceptible d'apprentissage et selon quelles modalités?

\section{La nature problématique du jugement esthétique}

3 En tant qu'elle est une forme d'attention au monde, la conduite esthétique nous met en relation avec des objets; elle est donc une forme de relation cognitive, mais qui devient le support d'une satisfaction. ${ }^{2}$ Cette conduite peut être adoptée vis-à-vis de tout objet naturel ou artificiel et pas seulement envers les œuvres d'art. Il faut distinguer entre l'appréciation esthétique (état de plus ou moins grande satisfaction) et le jugement esthétique (actequi attribue certains prédicats, beau, profond, sublime... à un objet), même si le second se fonde sur la première. Le jugement esthétique est un jugement de valeur, c'est-à-dire un jugement relationnel relatif aux intérêts qu'il exprime, et non un jugement de fait. Dire qu'un tableau a été peint par Poussin ou par Dughet est un 
jugement d'attribution (de fait); mais dire qu'il est beau, ou laid, est un énoncé esthétique d'ordre évaluatif. "Beauty is in the eye of the beholder" nous dit Hume, ${ }^{3}{ }^{c}$ 'est-àdire «La beauté est dans l'œil du spectateur ». Mais le jugement esthétique comporte un processus d'objectivation : je projette la valeur de satisfaction procurée dans ma relation à l'objet dans l'objet lui-même. Je transforme une propriété relationnelle en une propriété interne de l'objet. Le jugement esthétique est subjectif au sens où il s'appuie sur une expérience subjective singulière, mais il n'est pas obligatoirement individuel (l'intérêt exprimé par le jugement peut être collectif, partagé par plusieurs ou même par le plus grand nombre). Dire que l'appréciation esthétique est objectivante revient à souligner le fait qu'elle attribue à l'objet de sa satisfaction des propriétés qui sont censées rendre compte du plaisir éprouvé. Mais toute propriété « perceptuelle » des objets a-t-elle une pertinence esthétique? La délimitation des prédicats esthétique semble une tâche impossible sinon vaine. Naguère, Robert Blanché, en logicien sérieux avait tenté l'aventure dans un petit ouvrage: Des catégories esthétiques (Vrin, 1979) Le résultat n'est pas totalement convaincant. Au beau, on peut adjoindre le joli, le gracieux, le charmant, le pittoresque, le profond, l'équilibré, le majestueux, le sublime... La liste est inépuisable et toute tentative de classification peut encourir le reproche d'arbitraire.

Quant à la validité du jugement de goût, on peut schématiquement distinguer deux positions traditionnelles : celle qui prétend qu'il existe un beau idéal, des critères du beau, c'est-à-dire des règles régissant la production et l'appréciation des œuvres belles ; et celle qui prétend que l'expérience esthétique ne s'explique pas, ne se partage ni ne se communique : le jugement esthétique se réduirait alors à n'être qu'un jugement de goût purement sensible dans lequel le sujet manifesterait sa jouissance ou son agrément sans pouvoir en rendre compte et s'abîmerait dans une intimité instable et muette. Il est donc nécessaire de comprendre comment le jugement esthétique peut être à la fois subjectif et objectif, enraciné dans la particularité et ouvert à l'universel et comment une interprétation peut échapper à l'arbitraire sans toutefois prétendre à la scientificité.

Pour toute pensée classique, les formes du beau sont déductibles de principes rationnels. « Il appartient à l'esprit, c'est-à-dire à l'entendement, de juger de la beauté, parce que juger de la beauté, c'est juger de l'ordre, de la proportion et de la justesse. $»^{4}$ Il y a des lois de l'expression artistique (la règle des trois unités, pour le théâtre, les divers " canons " de la sculpture...). La nature, comme ordre, est un modèle à imiter; et, par là, le beau s'identifie au vrai. Mais dans cet idéal classique, le vrai n'est qu'une forme du vraisemblable et la convenance n'est souvent qu'un modèle social déterminé. Historiquement, le XVIII e siècle voit apparaître la notion de "goût » avec la subjectivation des phénomènes esthétiques. L'approche empiriste se penche sur la conscience sensible et le problème esthétique devient celui du goût; les variations de la sensibilité et du plaisir se substituent aux normes de la raison. Pour l'Abbé Du Bos, par exemple, le but de l'art est de toucher, il répond à un besoin d'émotion de l'âme en suscitant des passions. Il faut alors analyser les sentiments produits par les œuvres. Mais la subjectivité du jugement de goût pose le problème de la justification de l'accord sur certaines évaluations. On est alors contraint de postuler un «sens commun » à tout public ou une certaine uniformité de la « nature humaine».

6 En termes simples, le problème peut se formuler ainsi : comment le jugement esthétique, qui est singulier et exprime directement un sentiment individuel, peut-il réaliser une intersubjectivité (une entente entre plusieurs sujets) ? Comment le sentiment de plaisir et de déplaisir peut-il donner lieu à un jugement ayant une validité intersubjective (c'est-à- 
dire une certaine forme d'universalité) ? La question de la formation de la sensibilité esthétique dépend des réponses que l'on donne à ce problème. Notre objet est de montrer que le plaisir esthétique n'est pas un simple agrément ou une simple jouissance puisqu'il peut se cultiver et grandir dans la réflexion intellectuelle.

\section{La mesure du beau}

\subsection{Le privilège de la forme}

7 Il est très difficile de définir le beau. L'usage du terme dans le langage " ordinaire » est très extensible. On peut, toutefois, dégager une constante : est beau ce qui plaît par la forme (le latin dit «formosus»). La forme, c'est essentiellement et originellement la figure spatiale. La beauté est ainsi en relation étroite avec la géométrie, et plus particulièrement avec les figures de la géométrie grecque. Mais à quelle condition une forme spatiale est-elle belle ? Les psychologues de la Gestalt Théorie ont pensé la déceler dans la notion de la bonne forme: la belle figure est celle qui répond à des exigences de régularité, d'équilibre, de symétrie et de proportion qui rendent la forme intuitivement perceptible comme un tout unifié, où apparaissent des lois de construction. La forme c'est aussi, au sens aristotélicien, l'acte, l'accomplissement ; en ce sens, la beauté est forme en tant qu'achèvement, complétude.

Si la beauté est forme, elle est l'objet d'un spectacle ; elle est essentiellement scopique : «Le mot de beau semble plus particulièrement se rapporter au sens de la vue. ${ }^{5} \mathrm{~L} a$ vue est, dans la tradition philosophique, le sens le plus intellectualisé (avec l'ouïe). Il s'adresse à la forme du sensible et non à sa matière.

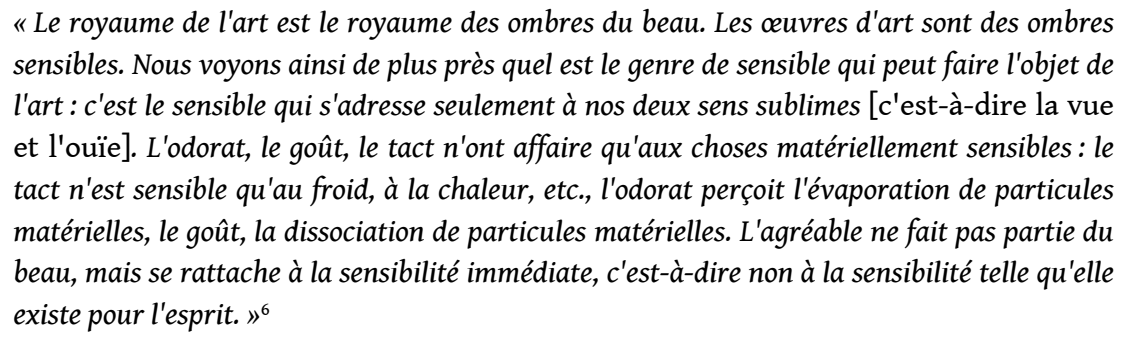

La chose belle tend à se réduire à une architecture abstraite. Mais si le beau est d'ordre purement intelligible, la pure beauté intelligible n'est plus d'ordre « esthétique », elle est hors du sensible. Dans la théorie des arts, le privilège de la forme engendre la primauté du dessin; et un artiste "platonicien» comme Léonard de Vinci peut déclarer que la peinture est «cosa mentale ». Dans les arts de la durée (musique et poésie) la domination de la forme se manifeste dans les constructions mélodiques ou harmoniques et dans les règles de versification (soit un exemple moderne : la musique dodécaphonique et sérielle inaugurée par Schoënberg est essentiellement une construction intellectuelle, du moins dans sa théorie).

\subsection{L'idéal grec}

Cette idée de beauté intelligible prend sa source en Grèce ancienne. Pour les anciens Grecs la nature est un cosmos. Celui-ci constitue un principe d'ordre et d'harmonie qui règle les rapports entre les différents êtres et qui définit les justes proportions entre les éléments constitutifs de chaque être, que ce soit une œuvre d'art, un organisme vivant ou 
une cité. L'ordre et la beauté de l'univers sont la manifestation non de relations de cause à effet, comme pour la science moderne, mais d'une justice divine. L'homme, parce qu'il est esprit, est le seul à pouvoir contempler l'ordre et l'harmonie du cosmos et développer son appartenance à ce cosmos. Mais pourquoi ne se contente-t-il pas d'une contemplation de la nature? A quelles fins répond la création d'œuvres belles?

11 L'homme est aussi l'être le plus inquiétant. Il est l'être le plus porté à s'écarter de son principe, à s'éloigner de son centre, à contester la loi naturelle d'équilibre. C'est par ce qu'il y a de plus terrible chez l'homme que la condition humaine se manifeste. C'est ce que Sophocle voulait traduire dans un vers d'Antigone: "Nombreuses sont les choses terribles, mais de toutes la plus grande c'est l'homme. » L'homme dépasse la juste mesure par la puissance qu'il rassemble en lui. Il est «terrible» par la démesure de sa volonté, par l'hybris (excès) qui le porte à mettre en danger l'ensemble des rapports qui constitue le cosmos qui lui est propre. La tragédie met en scène cette démesure: meurtres, massacres, parricides, incestes, défis aux dieux... Mais l'œuvre tragique, comme toute œuvre d'art, a pour fonction de rétablir l'équilibre. Ce que les grecs appellent la catharsis, c'est l'équilibre reconquis, c'est sentir que le cosmos éternel demeure le fondement inébranlable. Le modèle de cette « justice » cosmique c'est la proportion géométrique qui triomphe dans les arts plastiques et l'architecture. Ce qui fait la particularité des œuvres tragiques et leur ressort, c'est de susciter l'effroi afin de révéler l'aspect redoutable de cette justice.

12 Pour l'art grec, le nombre est la clef de l'harmonie. Un certain nombre de proportions remarquables, dont le célèbre nombre d'or, fournissent la base des arrangements formels. La spéculation mathématique s'étend au domaine des arts; les consonances musicales s'ordonnent autour de rapports déterminés: le diapason (1/2),le diapente (2/3)et le diatesseron (3/4)[aujourd'hui l'octave, la quinte et la quarte]. Les arts plastiques (par exemple, la frise du Parthénon) empruntent ces rythmes musicaux. Pour les temples, les dimensions de l'ensemble et des parties sont fonction d'une unité de mesure: le module, qui est, en général, le rayon (ou le diamètre) de la colonne à la base du fût. La bonne mesure -l'eurythmie - est la détermination des cotes en fonction du module. Dans l'art monumental, l'homme sert de mesure et d'échelle. Mais la représentation du corps humain est elle-même soumise à des canons. Le canon n'est pas une moyenne des proportions des parties du corps de divers individus, il est plutôt un idéal qui prétend à la vérité et donc à la stabilité. Cependant, l'art grec a connu une variation dans la détermination des canons : Lysippe soutenait que la dimension totale d'un corps humain idéal était huit fois celle de la tête, alors quePolyclète se contentait de sept! Les dimensions des figures sont liées aux lois de l'optique: la distance entre le regard et l'objet impose des déformations (à taille égale un personnage à petite tête paraitra plus grand qu'un personnage à grosse tête).

Le célèbre nombre d'or (autrement nommé, section dorée ou même «divine proportion ») a donné lieu à des analyses aux limites de l'irrationnel ${ }^{7}$. Il s'agit simplement d'une propriété mathématique remarquable. Pour obtenir cette proportion, on divise un segment de droite en deux segments $a$ et $b$, tels que $a / b=(a+b) / a ;$ on obtient $: a / b=(1$ $+\sqrt{5}) / 2=1,618 \ldots$ Dans l'histoire de l'art, les artistes ont souvent utilisé les propriétés de cette section pour leurs constructions formelles; l'architecte Le Corbusier en est un exemple récent. Mais de là à y voir le modèle absolu de la beauté pure calquée sur la structure du cosmos, il y a un pas que nous ne pouvons plus franchir ! Chez les peintres, le travail de «composition» du tableau s'ordonne largement autour de la construction de 
lignes de forces qui constituent les "charpentes $»^{8}$ de la représentation. Figures géométriques simples et proportions sont mobilisées à cet effet. On pourrait enfin voir dans l'école allemande du Bauhaus, le triomphe de cette conception «scientifique » de la beauté où la pureté de la forme rejoint la fonctionnalité rationnelle.

\subsection{Objections} Soulignant la nature surprenante de l'effet esthétique, l'abbé Du Bos écrivait: «On éprouve tous les jours que les vers et les tableaux causent un plaisir sensible ; mais il n'en est pas moins difficile d'expliquer en quoi consiste ce plaisir qui ressemble souvent à l'affliction, et dont les symptômes sont quelquefois les mêmes que ceux de la plus vive douleur. L'art et la poésie ne sont jamais plus applaudis que lorsqu'ils ont réussi à nous affliger. $»^{9}$ En opposition - ou en liaison - avec le sentiment d'harmonie, les hommes sont aussi fascinés par l'effroyable, le douloureux ou le terrible. Mais les Grecs n'avaient-ils pas déjà pressenti cette dualité à travers l'opposition des figures d'Apollon et de Dionysos? En deçà de l'équilibre et de la rationalité apollinienne, il y a toujours la puissance effrayante des instincts dionysiaques. A l'expérience du beau il faut ajouter le sentiment du sublime qui est d'une toute autre nature. Le sublime, contrairement à la beauté, est ce qui surpasse toute limite ; il ouvre une perspective sur l'infini. qualités sensibles des matériaux: le groupe "Support/Surface" en peinture, les accumulations et les expansions chez les sculpteurs, la musique "concrète", etc. L'histoire moderne des formes est aussi celle de l'informe. Les artistes introduisent des matériaux structurellement inconsistants. Duchamp inaugure les sculptures molles comme le «Pliant de voyage » (une housse de machine à écrire) et J. Beuys dispose des coulées de graisse sur des chaises... Le monument s'évanouit au profit du tas. Dans l'art contemporain, le sculpteur devient plasticien; la figuration et la représentation disparaissent pour laisser place à un hyper matérialisme. Ce que l'œuvre dévoile, ce n'est plus un sens mais les qualités mêmes de la matière : textures, striages, pliages, distorsions et éraillements. L'exaltation de la matière passe par la mort de l'objet à la forme clairement délimitée. description de l'expérience esthétique. 


\section{L'expérience esthétique}

\subsection{Une « physiologie » du beau et du sublime} mutuellement incompatibles: une expérience du sublime, dans laquelle le plaisir était moins évident que l'intensité d'une passion marquée du sceau de la terreur, et une expérience du beau qui se fondait sur l'amour, la communication et l'aisance relationnelle. En rompant avec la conception intellectualiste du beau identifié au bon et au vrai, il refuse de confiner la sensibilité esthétique dans le cadre des deux sens «nobles » : la vue et l'ouïe. C'est plutôt le sens tactile qui devient le modèle de tous les autres. La méthode de Burke est empiriste; il faut partir de l'expérience, comparer, dégager des constantes et l'on pourra déterminer les sources, la nature et les effets du beau et du sublime. "Une théorie fondée sur l'expérience et qui n'est pas purement hypothétique est toujours bonne pour ce qu'elle explique... $»^{10}$

Qu'est-ce que le goût? «Rien de plus que cette faculté ou ces facultés de l'esprit qui sont émues par les œuvres d'imagination et les arts raffinés, ou qui prononcent des jugements à leur endroit. $»^{11}$ Le goût n'est pas une faculté séparée, un sixième sens ou une sorte d'instinct, mais un rapport des sens, de l'imagination et du jugement. Tous les hommes étant dotés des mêmes sens, il y a un accord fondamental sur les déterminations sensorielles (le vinaigre est acide et le miel doux), mais aussi sur les appréciations (la douceur est plaisante et l'acidité déplaisante). Si, par delà ce goût naturel, coexistent des goûts acquis, ceux-ci sont toujours rapportés aux premiers. "Ainsi le plaisir de tous les sens... est semblable chez tous les hommes, grands ou petits, savants ou ignorants. $»^{12}$ L'imagination, toujours reproductrice, engendre un plaisir spécifique qui repose sur la ressemblance. Dès lors les images qui nous plaisent sont celles qui reproduisent des objets plaisants; et l'accord des imaginations est à l'image de celui des sens. Le postulat d'une uniformité de la nature humaine fonde la communauté des principes du goût. Mais alors, d'où proviennent les variations? Pour Burke, la cause en est simplement les divers niveaux de sensibilité naturelle et le degré d'attention plus forte ou plus soutenue que l'on porte à l'objet. Plaisir et douleur, comme éléments simples, sont le point de départ de toute appréciation esthétique qui se départage en deux types d'expériences : le sublime et le beau.

Le sublime a sa source dans la douleur, la peur et le terrible : «Tout ce qui est propre à susciter d'une manière quelconque les idées de douleur et de danger, c'est-à-dire tout ce qui est d'une certaine manière terrible, tout ce qui traite d'objets terribles ou agit de façon analogue à la terreur, est source du sublime, c'est-à-dire capable de produire la plus forte émotion que l'esprit soit capable de ressentir. $»^{13}$ Le danger et la douleur, lorsqu'ils sont éprouvés fortement dans notre chair, ne provoquent pas d'expérience esthétique. Ils ne sont «délicieux » qu'à distance et avec certaines modifications. Le délice, c'est la sensation qui accompagne l'éloignement de la douleur ou du danger. Burke distingue trois types de phénomènes qui suscitent la terreur et, par delà, le sublime : l'obscurité (la nuit, l'atmosphère sombre des grottes et des temples, l'obscurité des forêts immenses suscitent des émotions et des passions fortes), le pouvoir (qu'il soit de la nature, des princes ou de Dieu, le pouvoir, par sa force, induit la terreur; la majesté est toujours redoutable et provoque la crainte respectueuse qui ôte tout usage des facultés), la vacuité 
, le vaste et l'infini (des dimensions de l'espace, c'est la profondeur qui nous frappe le plus et l'infiniment grand comme l'infiniment petit perdent l'imagination). L'infini a tendance à remplir l'esprit de cette sorte d'horreur délicieuse qui est l'effet le plus authentique et le meilleur critère du sublime. Le grandiose a donc pour fondement la terreur qui, lorsqu'elle est modifiée, cause cette émotion de l'esprit que Burke nomme l'étonnement. Douleur et terreur modifiées de manière à n'être pas réellement nocives sont capables de donner du délice, une sorte de tranquillité teintée de terreur qui est une des passions les plus fortes.

21 Le beau: «Par beauté, j'entends cette qualité ou ces qualités des corps, qui leur permettent d'exciter l'amour ou une passion voisine ${ }^{14}$. Mais il faut prendre soin de bien distinguer l'amour du désir, énergie propre à l'esprit qui l'entraîne à posséder des objets qui ne l'affectent pas obligatoirement par leur beauté. Burke commence par rejeter les principes classiques du beau qui ne réside ni dans la proportion ni dans la convenance. L'idée de beauté ne relève pas de la mesure et n'a que faire du calcul et de la géométrie ; pire même, l'excès de proportionnalité peut lui être néfaste. La nature offre des exemples frappants de disproportionnalité que nous apprécions cependant: la rose et sa tige, le pommier et ses fleurs ont des proportions inverses et l'anatomie animale témoigne d'une très grande diversité. Quant aux canons de la beauté humaine, ils ne s'accordent pas entre eux et se rencontrent aussi chez les gens laids! «Or si l'on accorde que la beauté est compatible avec des formes et des arrangements très différents et même contraires, on doit en conclure qu'aucune mesure déterminée, agissant d'après un principe naturel, n'est nécessaire pour l'engendrer. $\aleph^{15}$ La convenance, c'est-à-dire l'adaptation d'un élément à sa fin, n'est pas plus un principe de beauté. Les idées d'utile et de beau sont dissemblables. Bien des choses utiles ne sont pas belles et inversement, on ne saurait attribuer une quelconque utilité à des choses fort belles. «L'effet de la proportion et de la convenance, pour autant, du moins, qu'elles procèdent du pur examen de l'œuvre ellemême, produit l'approbation et l'assentiment de l'entendement, non l'amour ou une passion voisine. $»^{16}$

Quelles sont alors les qualités sensibles à l'origine du sentiment de beauté ? Trois déterminations émergent de l'analyse de Burke: la petitesse, la «smoothness» et la variation progressive. L'amour est attiré par les objets petits et agréables; nous aimons ce qui se soumet à nous. La « smoothness » (le lisse) est cette qualité à laquelle la beauté doit la part essentielle de son pouvoir. Elle est à la fois l'égalité d'une surface ou son absence d'aspérité, la douceur du goût, la grâce des manières et la suavité du style : feuilles lisses des arbres et des fleurs, pentes douces des jardins, surfaces unies des eaux ornant le paysage, pelage uni des oiseaux et des animaux, peau lisse des femmes, surfaces lisses et polies de divers meubles décoratifs. Quant à la variation progressive, elle est la sensation de continuité sans rupture. La sensation de beauté n'est provoquée ni par les lignes droites ni par les tracés anguleux, mais par les parcours en déviation continuelle et progressive. Les corps dont la surface varie continuellement sont les plus agréables ou les plus beaux au toucher. Dans le mouvement, c'est l'oscillation douce, dont le paradigme est la balançoire, qui procure le plaisir le plus proche de la beauté. Avec Burke, l'esthétique du beau est celle de la tendresse : la douceur est le goût du beau. Si l'effet du sublime est l'étonnement, celui du beau est la détente : «Qui ne connaît ces expressions communes à tous les temps et à tous les pays par lesquelles on se dit amolli, relâché, énervé, dissous, fondu de plaisir ? $»^{17}$ 
'entreprise de Burke est une archéologie de nos passions esthétiques, elle prétend montrer comment l'appréhension de qualités sensibles va de pair avec un investissement d'ordre à la fois sensuel, imaginatif et intellectuel. Mais la description des sources et des effets ne dit rien de l'évolution du goût ni de son éventuel apprentissage.

\subsection{La culture du goût}

(ici Hume) constate qu'il est vain de discuter des goûts, mais il existe une espèce de sens commun qui s'oppose à lui : "Quelque différents que puissent être les goûts des hommes, leur raisonnement général sur ces sujets est communément le même. $\aleph^{18} \mathrm{Il}$ est impossible d'affirmer l'équivalence des œuvres; nous établissons des hiérarchies de grandeur. Cela ne veut pas dire que tous les hommes reconnaissent effectivement la même hiérarchie, mais que nous recherchons naturellement des règles. Ces règles ne sont pas d'ordre scientifique, mais trouvent un fondement dans l'expérience du plaisir. « Les règles ne sont pas autre chose que des observations générales concernant ce qui a plu universellement dans tous les pays et à toutes les époques. ${ }^{19}$. Le goût est une opération complexe et délicate qui requiert beaucoup de conditions favorables. Faute de ces conditions, l'exercice normal du goût est perturbé et il est impossible de reconnaître la beauté là où elle est. C'est pourquoi la beauté et ses règles ne peuvent apparaître qu'à un certain point de vue général qui élimine, en quelque sorte statistiquement, les possibilités d'une telle perturbation. Il y a donc bien une beauté universelle. Elle correspond à une relation établie par la nature.

La culture du goût va donc consister à instaurer en soi les circonstances de son exercice naturel. La première condition est l'état sain des organes ; la seconde est la " délicatesse » du goût, c'est-à-dire la perception des détails et la précision des nuances. Pour illustrer cette délicatesse, Hume utilise ce qu'on pourrait nommer : le paradigme de Sancho. Dans l'essai De la norme du goût, il rapporte l'anecdote du Don Quichotte où Sancho raconte que deux de ses parents, appelés pour goûter un vin et juger s'il était bon, le boivent, le trouvent bon, mais remarquent, l'un un petit goût de cuir, l'autre un petit goût de fer. On se moque d'eux et l'on fait remarquer la contradiction de ces points de vue. Mais lorsqu'on vide le tonneau, on trouve, dans le fond, une vieille clé attachée à une courroie de cuir ! Il y a une analogie entre le goût physique et le goût esthétique. Les parents de Sancho perçoivent une qualité très subtile que les autres ne sont pas capables de distinguer. Le goût esthétique suppose lui aussi cette distinction fine des qualités. « Là où les sens sont assez déliés pour que rien ne leur échappe et en même temps assez aiguisés pour percevoir tout ingrédient introduit dans la composition: c'est là ce que nous appellerons délicatesse de goût que nous employions ces termes selon leur sens littéral ou leur sens métaphorique. $»^{20}$ (Mais dans cet apologue, Hume ne distingue pas entre le jugement de fait qui discrimine les goûts de fer et de cuir et le jugement de valeur qui apprécie le bon ou le mauvais vin. Les sens aiguisés des parents de Sancho les rendent-ils plus aptes à déterminer la valeur du vin? L'identification des saveurs n'est pas encore un jugement esthétique.)

26 Cette délicatesse est un don de la nature, mais inégalement distribué. Tous les hommes n'ont pas les mêmes aptitudes à discerner les qualités instituées par la nature pour nous plaire. Toutefois, cette délicatesse naturelle peut toujours être développée par l'exercice. Hume expose quatre moyens principaux pour développer le goût: la pratique d'un art particulier et l'étude répétée d'un genre de beauté permettent de distinguer chaque 
partie, ses qualités, sa fonction et, en précisant l'appréciation, l'affinent et l'affermissent ; la fréquentation répétée des œuvres nous incite à dépasser la première impression, à éviter la précipitation, à ne pas se laisser séduire par une beauté seulement superficielle ; par la comparaison des différentes sortes de beauté, l'homme de goût acquiert l'expérience qui lui permet de disposer les différentes beautés selon l'ordre de leur perfection croissante; enfin, l'examen de ses propres préjugés évite toute mauvaise anticipation. Chaque œuvre a ses propres exigences, des règles particulières; elle impose un point de vue qu'elle définit elle-même. Ne pas adopter ce point de vue, c'est avoir des préjugés, c'est considérer l'œuvre de telle sorte qu'il est impossible de goûter sa beauté propre. "Un homme sous l'empire du préjugé garde avec obstination sa position naturelle sans se placer à ce point de vue précis que l'œuvre demande. $»^{21} \mathrm{Il}$ y a donc toute une part de réflexion qui consiste à déterminer le genre de l'œuvre, les conditions de son style, les circonstances de son élaboration afin de déterminer le point de vue sous lequel l'œuvre doit être considérée. C'est le travail du bon sens et de la raison. Le goût n'est pas la raison, mais la présuppose.

Pour bien juger des œuvres, il faut donc à la fois une expérience des arts, des beautés déjà produites, un entendement sain et des qualités intellectuelles. Ces qualités ne se rencontrent qu'assez rarement chez un même homme : «Bien que les principes du goût soient universels et presque, sinon entièrement les mêmes chez tous les hommes, cependant bien peu d'hommes sont qualifiés pour donner leur jugement sur une œuvre d'art ou pour établir leur propre sentiment comme étant la norme de la beauté. $»^{22}$ Parce qu'il est une heureuse synthèse de dons naturels et d'exercices, l'expert est à la fois source de normes et éducateur; il indique aux autres les chemins de l'exercice du goût naturel. Mais comment reconnaître l'excellence du critique? C'est une question de fait: si un individu nous paraît doué de délicatesse, d'expérience, de bon sens et s'il est libre de tout préjugé, on ne pourra nier sa compétence. La norme du goût n'est pas une possibilité introuvable, elle existe de fait, elle est connue et reconnue, elle guide l'exercice de notre goût. Il y a une permanence de l'œuvre d'art qui atteste non seulement l'existence de normes mais qui montre comment ces normes déterminent effectivement la composition des œuvres et les appréciations des hommes.

Mais il reste à rendre compte de la persistance de la variété des appréciations esthétiques. Hume évoque plusieurs raisons: l'imperfection du goût des hommes ordinaires, les différentes humeurs de chaque homme en particulier, les mœurs et les opinions particulières à chaque âge et à chaque pays. Pour expliquer le fait que les évaluations peuvent être partagées, la philosophie de tradition empiriste postule des «constantes anthropologiques ", mais celles-ci restent à préciser et ne sont peut-être qu'une autre manière de nommer la «nature humaine ». Faut-il également recourir à la nature sociale de l'existence humaine? L'appartenance à un groupe détermine une adhésion à des valeurs communes. Mais le propre de tout groupe est de se différencier d'autres groupes par les conduites comme par les valeurs.

Il y a des normes du goût, c'est un fait. L'empiriste se refuse à aller au-delà de ce fait. Mais comment un jugement de goût est-il possible ? Quel est le fondement de sa validité ? Seule une critique du jugement esthétique peut répondre. 


\section{Analytique du jugement de goût}

La faculté de juger est le pouvoir de penser le particulier comme compris sous l'universel. Mais à partir de là, on peut distinguer deux types de jugement : le jugement déterminant qui, à partir d'une règle, d'une loi ou d'un principe universels comprend le particulier, et le jugement réfléchissant qui, partant d'un particulier donné, doit chercher l'universel. Le jugement de goût appartient à cette dernière catégorie. Si l'on suit Kant, ${ }^{23}$ quatre caractéristiques fondamentales en découlent.

\subsection{La satisfaction désintéressée}

Le jugement de goût n'est pas un jugement de connaissance, il ne nous fait rien connaître de l'objet; il est esthétique, c'est-à-dire purement subjectif en ce qu'il renvoie au sentiment de plaisir ou de peine du sujet. De surcroît, le goût est désintéressé ; il est indépendant de l'intérêt que je peux porter à l'existence d'une chose. Par exemple, trouver beau un édifice ne dépend pas du jugement que je peux porter sur son utilité, sa fonction ou sur le besoin que j'en puis avoir. Mon attitude est de simple contemplation. Dès lors, le beau se distingue clairement de l'agréable et du bon qui, en tant que dépendants de la faculté de désirer, sont intéressés à l'existence des objets. «L'agréable signifie pour chacun ce qui lui fait plaisir; le beau ce qui simplement lui plait; le bon ce qu'il estime, ce qu'il approuve, c'est-à-dire ce à quoi il attribue une valeur objective. $»^{24}$ L'agréable est une jouissance purement sensorielle qui suppose la possession de l'objet, son appropriation par le désir (l'exemple culinaire vient immédiatement à l'esprit). La qualité de bon est attachée à quelque chose en tant qu'utile (bon à...) ou bon en soi (valeur morale); elle suppose une connaissance, un concept de la chose pour pouvoir être attribuée. Le jugement de goût, quant à lui, est libre et désintéressé; le sujet qui le profère est dans une attitude de «libre faveur » vis-à-vis de l'objet, ni asservi au désir sensible de la chose (l'agréable), ni au désir rationnel de l'action (le bien). Si dans le besoin l'homme est asservi à la nature, le goût ne peut intervenir que lorsque le besoin est apaisé, comme il doit être indépendant des intérêts et des obligations de la morale.

\subsection{L'universalité sans concept}

Ce qui est beau ne l'est pas simplement pour moi (comme l'agrément), mais lorsque je dis d'une chose qu'elle est belle, j'attribue aux autres le même plaisir. Je parle de la beauté commesi c'était une propriété de la chose. Cette universalité n'est pas une question de fait, elle ne résulte pas du constat des convergences des goûts individuels (comme le voulait l'empirisme), elle est plutôt une exigence. Le sujet qui juge d'une beauté prétend à l'assentiment de tous, mais il est hors d'état de le démontrer parce que le jugement de goût n'est pas un jugement de connaissance qui repose sur le concept de la chose.

"Il faut donc bien voir ici que, dans le jugement de goût, rien n'est postulé que cette universalité des voix en ce qui concerne le plaisir, sans la médiation des concepts: par conséquent, on postule uniquement la possibilité d'un jugement esthétique qui puisse en même temps être considéré comme valant pour chacun. Le jugement de gout lui-même ne postule pas l'adhésion de chacun (car seul peut le faire un jugement logiquement universel, capable d'alléguer des raisons); il ne fait que prêter à chacun cette adhésion, comme un cas 
de la règle dont il attend la confirmation non de concepts, mais de l'adhésion des autres.

L'universalité des voix n'est donc qu'une idée. ${ }^{25}$ aptitude à communiquer. Ce plaisir universellement communicable c'est celui éprouvé au libre jeu de l'imagination et de l'entendement.

\subsection{La finalité sans représentation d'une fin}

Nous avons vu que l'objet beau retient notre contemplation, c'est-à-dire qu'il ne nous incite ni à la connaissance, ni à l'action; nous n'en formons pas de concept, nous ne nous le proposons pas comme fin de notre action; nous restons enfermés dans un sentiment subjectif (quoique communicable). Mais reste à se demander si l'objet beau est conforme à une fin. Il y a deux sortes de finalités : externe et interne. La finalité externe d'un objet est sa conformité à sa fonction; c'est son utilité. La finalité interne est la conformité d'un objet à ce qu'il doit être, à son concept, c'est sa perfection. Or, le jugement de goût étant désintéressé, le beau ne peut être confondu avec l'utile. Mais la beauté ne s'apparente pas plus à la perfection. Pour se représenter la finalité objective d'une chose, le concept de cette chose doit être préalablement possédé ; or, le jugement de goût ne repose pas sur le concept de la chose. Une telle analyse exclut tout modèle mathématique de la beauté. La forme géométrique n'est pas belle en soi, car le beau c'est l'au-delà de la règle ; et il est impossible de définir une règle objective du goût permettant de déterminer ce qui est beau. L'irrégularité baroque est aussi belle que la régularité mathématique. Le beau ne se ramène à aucune fin matérielle; dans le jugement de goût n'apparaît qu'une finalité formelle et subjective. Est beau l'objet qui répond à une fin ou à une intention dont nous ne pouvons déterminer le concept, que nous ne pouvons nous représenter. Sa finalité reste indéterminée. « La finalité peut donc être sans fin, dès lors que nous ne situons pas les causes de cette forme dans une volonté, mais que, néanmoins, nous ne pouvons nous rendre concevable l'explication de sa possibilité qu'en la dérivant d'une volonté. $»^{26}$

Toutefois, Kant distingue deux types de beauté : la beauté libre et la beauté adhérente. «La première ne suppose nul concept de ce que doit être l'objet; la seconde suppose un tel concept, ainsi que la perfection de l'objet par rapport à ce concept. $»^{27} \mathrm{Il}$ y a beauté adhérente, par exemple, lorsqu'en jugeant de la beauté d'un édifice, je prends en considération la fin à laquelle il est destiné (église, palais, arsenal, maison de campagne, etc.) ; de même d'un cheval selon que je sais qu'il est «de trait » ou "de course »; de même de l'homme, de la femme, de l'enfant, dans la mesure où l'on aura la représentation conceptuelle de ce qu'ils doivent être selon le sexe et l'âge (virilité, féminité, puérilité). Kant met l'accent sur l'infériorité, au point de vue de la pureté, de cette beauté adhérente relativement à la beauté libre où la fin est ignorée, au double sens de ne pas savoir et de ne pas vouloir savoir: ainsi les fleurs sont des libres beautés de la nature si on fait abstraction de leur finalité reproductrice, ainsi que les plumages éclatants des oiseaux. Dans les œuvres humaines, Kant cite, comme exemples de beautés libres, des motifs décoratifs : les lignes grecques, les rinceaux, les motifs des tapisseries qui ne signifient rien, ne représentent rien. Certains ont souligné qu'il y avait là une justification avant la lettre de l'art abstrait du XXe siècle qui vise l'abandon de la représentation. La musique sans texte et les fantaisies sont aussi à ranger dans les beautés libres. L'adhérence est donc une servitude par rapport au pur jugement de goût. Mais cette distinction n'est pas une opposition. La beauté adhérente est, d'une certaine manière, une beauté 
« appliquée ». La satisfaction esthétique peut pactiser avec la satisfaction intellectuelle. Par exemple, dans la représentation picturale d'un cheval de course, je peux apprécier la beauté libre des formes et des couleurs et les qualités « adhérentes » qui tiennent à ce que doit être un coursier : courbe du poitrail, longueur de l'encolure, etc.)

\subsection{La satisfaction nécessaire} nécessaire à la satisfaction. Mais cette nécessité n'est pas objective ; l'adhésion de tous ne peut reposer que sur la postulation d'un sens commun. Mais il ne s'agit pas, pour Kant, de fonder ce sens commun sur l'expérience (à la différence de Hume); il n'est que la condition de possibilité du jugement de goût.

«Ce sens commun ne peut, à cette fin, être fondé sur l'expérience; car il entend légitimer des jugements qui contiennent un devoir: il ne dit pas que chacun s'accorderaavec notre jugement, mais que chacun doit être d'accord avec celui-ci. Ainsi le sens commun, dont je fournis ici comme exemple mon jugement de goût, en lui donnant pour cette raison une valeur exemplaire, correspond-il à une simple norme idéale dont la présupposition pourrait permettre à bon droit d'établir comme règle, pour chacun, un jugement qui s'accorderait avec elle et la satisfaction qui, relative à un objet s'exprimerait dans ce jugement. ${ }^{28}$ d'entendement. Mais contrairement au jugement de connaissance où l'entendement commande à l'imagination, dans le goût la liberté de l'imagination est première et la régularité de l'entendement lui est soumise, l'accord des deux facultés n'est que subjectif ; il s'agit, paradoxalement, d'une légalité sans loi.

\subsection{Au-delà de l'esthétique : le sublime}

«Est sublime ce qui, du fait simplement qu'on puisse le penser, démontre un pouvoir de l'esprit qui dépasse toute mesure des sens ${ }^{29}$. Le sublime c'est ce qui est absolument grand, c'est-à-dire ce qui est grand au-delà de toute comparaison. La grandeur du sublime surpasse toute limitation et toute mesure ; il ouvre une perspective sur l'infini. Dès lors le sublime ne doit pas être recherché dans les choses de la nature (ni les montagnes, ni les forêts, ni la mer déchaînée), mais dans les idées. En rompant avec la perspective de Burke, la notion de sublime, chez Kant, sort du domaine de l'esthétique pure pour introduire dans celui de la morale. « Tout affect relevant du genre courageux (celui qui éveille en nous la conscience des forces qui nous permettent de vaincre toute résistance) est esthétiquement sublime. ${ }^{30} \mathrm{Le}$ sublime est une disposition d'esprit qui réside dans le sentiment de l'impuissance de l'imagination à se représenter les idées de la raison; il est suscité par le conflit de la raison et de l'imagination; il s'agit d'un "plaisir négatif ». Le sublime est la satisfaction esthétique qui accompagne nos idées (notamment morales) ; il " élargit l'âme ». Il nous prépare à estimer quelque chose contre notre intérêt sensible ; il est donc le témoignage, en l'homme, de la tension du sensible vers le suprasensible.

Avec Kant, l'expérience esthétique se présente comme autonome, c'est un événement régi par sa propre loi, indépendant d'autres types d'expériences ou de discours comme la connaissance ou l'évaluation morale. Dans l'expérience esthétique, le plaisir est « libre »: des objets laids ou tragiques peuvent causer une jouissance esthétique, parce qu'il ne s'agit justement pas du plaisir pris à ces objets eux-mêmes, c'est-à-dire tels qu'ils peuvent être perçus et décrits en dehors de l'expérience esthétique. La différence esthétique se 
manifeste de façon exemplaire à travers le fait que nous pouvons, dans l'élément de l'expérience esthétique, tirer du plaisir de choses qui, en dehors de cet élément, ne nous causerait que du déplaisir. Mais l'aspect désintéressé du jugement esthétique et le postulat d'un «sens commun » restent des preuves fragiles de l'universalité du goût. Il est douteux que cette prétention soit vraiment démontrable.

\section{L'esthétique négative : du sensible au sens} artistique, son modèle reste essentiellement le beau «naturel » car les œuvres d'art ne constituent que des beautés "adhérentes ». Or, dans l'art, l'expérience esthétique ne se limite pas à une jouissance sensible spécifique, elle est aussi dotée d'un potentiel critique vis-à-vis de l'ordre social. C'est cette négativité de l'expérience esthétique, comme fonction critique que l'art exerce sur la réalité extérieure, que le philosophe Adorno s'est attaché à mettre en valeur. ${ }^{31}$

41 Pour Adorno, l'expérience esthétique n'est vraiment elle-même que lorsqu'elle se débarrasse de la jouissance. La théorie kantienne arrachait déjà le sentiment esthétique des désirs immédiats, mais elle restait pauvre vis-à-vis du phénomène artistique. La notion de satisfaction y demeurait indéfinie. Par la jouissance, le plaisir esthétique vise une réconciliation avec le monde ; mais il peut être aussi l'ignorance - ou pire, le masque de la souffrance et de l'aliénation. Le pur désintéressement fait de l'œuvre d'art un bien culturel agréable, un simple objet de consommation. L'art devient un délassement, un loisir qui compense l'aliénation économique et politique. « Le bourgeois désire que l'art soit voluptueux et la vie ascétique ; le contraire serait préférable. $\rrbracket^{32}$ Le plaisir esthétique éprouvé dans la rencontre avec les œuvres d'art comporte une composante négative qui réside dans l'expérience de la « dissonance ».

\subsection{L'industrie culturelle}

La réduction de l'expérience artistique à la pure sensualité est le fait de l'industrie culturelle. Par cette expression, Adorno entend l'exploitation systématique et programmée des biens culturels à des fins commerciales. Dans cette sphère, l'œuvre d'art ne fait que remplir un désir social. L'univers moderne de l'industrie culturelle se caractérise par son aspect unitaire: les films, les émissions radiophoniques ou télévisuelles, les magazines constituent un système qui confère à tout un air de ressemblance. La standardisation et la production en série répondent à des techniques de production et de reproduction. Le "cliché" devient l'ossature de base de toute production médiatique. Le mode de consommation du culturel est l'amusement, et s'amuser signifie ne penser à rien, oublier la souffrance même là où elle est montrée. Il s'agit d'une forme d'impuissance, de fuite, non pas devant la réalité, mais devant la volonté d'une résistance que cette réalité peut encore avoir laissé subsister en chacun. Les rapports de l'individu à l'œuvre s'inversent : une expérience esthétique authentique suppose que le sujet s'oublie lui-même devant l'œuvre (ce que Kant nommait : la « libre faveur »), qu'il se rende semblable à l'œuvre; alors que dans le monde de l'industrie culturelle, on tend à rendre l'œuvre semblable à soi. Le consommateur projette ses émotions sur ce qui lui est présenté ; l'œuvre n'est plus que le véhicule de la psychologie du spectateur. 


\subsection{La négativité} réconciliation, l'art, comme expression de la liberté, ne saurait oublier la souffrance: «Que deviendrait l'art en tant qu'écriture de l'histoire, s'il se débarrassait du souvenir de la souffrance accumulée? $\aleph^{33}$ Contre l'industrie culturelle, Adorno plaide pour toute œuvre de rupture qui est aussi une volonté d'émancipation. La teneur de vérité de l'art réside dans son aspect social. Mais l'art n'est social ni à cause du mode de sa production dans laquelle se concentre la dialectique des forces productives et des rapports de production, ni par l'origine sociale de son contenu thématique; il le devient beaucoup plus par la position antagoniste qu'il adopte vis-à-vis de la société, et il n'occupe cette position qu'en tant qu'art autonome. L'art ne se maintient en vie que par sa force de résistance sociale ; mais ce qui est social est son mouvement immanent contre la société et non sa prise de position manifeste. Ainsi, dans la musique dodécaphonique de Schönberg, les contradictions sociales apparaissent dans le matériau musical: «Les dissonances qui effraient (les auditeurs) leur parlent de leur propre condition; c'est uniquement pour cela qu'elles leur sont insupportables. $»^{34} \mathrm{Ou}$ encore :

«La force de la négativité dans l'œuvre d'art donne la mesure de l'abîme qui sépare la praxis du bonheur. Kafka n'éveille certainement pas la faculté de désirer. Mais la peur du réel qui répond aux pièces en prose de la 'Métamorphose' ou de 'La colonie pénitentiaire' , le choc, l'aversion et la nausée qui, secouant la nature, ont, en tant que réactions de défense, plus à faire avec le désir qu'avec l'ancien désintéressement qu'on lui réservait, à lui et à ses successeurs. $\|^{35}$

Ce qui est décisif dans les œuvres d'art sur le plan social revient à la part de contenu qui s'exprime dans leurs structures formelles. Ainsi, l'œuvre de Kafka est bien plus forte que tout roman qui traiterait de la corruption des trusts industriels. L'œuvre d'art n'est pas une simple célébration, mais aussi - et surtout - une protestation ; elle dénonce la fausseté étroite et bornée de l'existence pratique. Les sentiments d'harmonie et de sérénité, dans l'art, ne sont jamais purs: «L'art n'exprime l'inexprimable, l'utopie, que par l'absolue négativité de cette image. ${ }^{36} \mathrm{~L}$ 'expérience esthétique propre aux œuvres d'art ne se réduit pas à un plaisir pur, elle est aussi la conscience d'une impossible conciliation de l'homme et du monde car les œuvres d'art ne sont jamais ce qu'on voudrait qu'elles soient et démentent à chaque instant ce qu'elles voudraient être. La beauté n'est, selon la belle formule d'Adorno, que « la promesse du bonheur qui se brise.».

\section{Conclusion}

La sensibilité esthétique est condamnée au déchirement entre l'exigence de communicabilité universelle et la variété de ses formes, de la calme sérénité à l'effroi. Elle ne se confond ni avec le pur plaisir sensuel (l'agréable) ni avec la pure intelligence des formes. Si elle est désintéressée, ce n'est pas au point de nous couper de notre insertion sociale. «Celui qui jouit concrètement des œuvres d'art est un ignorant. Des expressions comme régal pour l'oreille lui suffisent. Mais si toute trace de jouissance était extirpée se poserait alors la question embarrassante de savoir pourquoi les œuvres d'art sont là. En réalité, plus on y comprend quelque chose, moins on jouit des œuvres. $»^{37}$ 
Que l'art ait abandonné le monde de la belle apparence, il y a là un acquis irrémédiable depuis l'apparition des avant-gardes. Le beau est passé du côté de la technique industrielle, du design et du fonctionnalisme. La grande série, reproductible à l'infini, dans l'architecture de fer et de verre, dans l'automobile ou l'avion, dans le mobilier, peut avoir sa beauté par l'adaptation des formes aux fonctions. Débarrassé de la beauté, l'art peut s'adonner à la signification provocante; en lui peuvent se manifester la dissonance et l'irréconciliation, mais c'est à condition de faire du sentiment esthétique une expérience de l'effroi plus que de l'harmonie. Devant les corps déformés des mises en scènes cruelles de Francis Bacon, au spectacle troublant de la violence de l'« Orange mécanique » de Stanley Kubrick, la fascination et la répulsion composent l'expérience esthétique moderne.

\section{BIBLIOGRAPHIE}

ADORNO T., Théorie esthétique, Paris, Klincksieck, 1974.

BLANCHE R., Des catégories esthétiques, Paris, Vrin, 1979.

BOULEAU C., Charpentes, la géométrie secrète des peintres, Paris, Le Seuil, 1963.

BURKE E., Recherche philosophique sur l'origine de nos idées du sublime et du beau, Paris, Vrin, 1990.

GENETTE G, L'œeuvre de l'art, Tome 2, La relation esthétique, Paris, Seuil, 1997.

GHYKAM., Le nombre d'or, Paris, Gallimard, 1959.

HUME D., Essais esthétiques, Paris, Vrin, 1974.

KANT E., Critique de la faculté de juger, Paris, Aubier, 1995.

SAINT-GIRONS B., Esthétiques du XVIII ${ }^{e}$ siècle, le modèle français, Paris, Sers éditeur.

SCHAEFFER J.-M., Les célibataires de l'art, Paris, Gallimard, 1996.

\section{NOTES}

1. SERRES M., Les cinq sens, Grasset, 1985. p. 23.

2. Sur ce point, voir : SCHAEFFER J.M., Les célibataires de l'art, Gallimard, 1996 ; notamment le chap. II.

3. HUME D., « De la norme du goût ", in Essais esthétiques II, Vrin, p. 82.

4. BOSSUET, Connaissance de Dieu et de soi-même, I, 8.

5. DESCARTES R., Lettre à Mersenne. 18 mars 1630.

6. HEGEL F.W., Esthétique, introduction, Éd. Aubier, p. 92.

7. $c f$. : GHYKA M.C., Le nombre d'or, Gallimard.

8. $c f$. : BOULEAU C., Charpentes. La géométrie secrète des peintres, Le Seuil.

9. DU BOS ABBÉ, Réflexions critiques sur la poésie et sur la peinture, 1719, I, p. 12.

10. . BURKE E., Recherche philosophique sur l'origine de nos idées du sublime et du beau, Vrin, 1757, p. 53. 
11. ibid., p. 57.

12. ibid., p. 60.

13. ibid., p. 80.

14. ibid., p. 133.

15. ibid., p. 138.

16. ibid., p. 150.

17. ibid., p. 192.

18. HUME D., « De la simplicité et du raffinement dans l'art d'écrire », in Essais Esthétiques, II, Vrin. p. 76.

19. HUME D., De la norme du goût, ibid., p.84.

20. ibid., p. 88.

21. ibid., p. 93.

22. ibid., p. 95.

23. KANT E., Critique de la faculté de juger, première partie: critique de la faculté du juger esthétique.

24. ibid., Éd. Aubier, Trad. A. Renaud, p. 188.

25. ibid., p. 194.

26. ibid., p. 199.

27. ibid., p. 208.

28. ibid., p. 219.

29. ibid., p. 232.

30. ibid., p. 256.

31. ADORNO T., Théorie esthétique, Éd. Klincksieck.

32. ibid., p. 25.

33. ibid., p. 344.

34. ADORNO T., Philosophie de la nouvelle musique, Gallimard, p. 30.

35. ADORNO T., Théorie esthétique, p. 24.

36. ibid., p. 51.

37. ibid., p. 24.

\section{RÉSUMÉS}

Qu'est-ce qu'un jugement de goût? Peut-il être fondé ? Comment le sentiment de plaisir et de déplaisir peut-il donner lieu à un jugement ayant une validité intersubjective? A quelles conditions un apprentissage du jugement de goût est-il possible? Si l'idée de beauté intelligible des Grecs s'avère insuffisante, c'est dans une perspective empiriste que l'on peut saisir ce que peut être une éducation du goût. La sensibilité esthétique est condamnée au déchirement entre l'exigence de communicabilité et la variété de ses formes; malgré son désintéressement, elle n'est pas coupée de toute insertion sociale.

What is an aesthetic judgment? On what can the judgment be founded? How can the feeling of pleasure or displeasure lead to a judgment that will have an intersubjective validity? Under what conditions is an apprenticeship in aesthetic judgment possible? If the notion of intelligible beauty of the Greeks proves to be inadequate we should turn to the empirical approach to grasp what an education in taste can be. Aesthetic sensibility is bound to be torn between the demands 
of communicability and the variety of its forms. Although it would seem to be disinterested, it is not cut off from all forms of social integration.

INDEX

Mots-clés : beau, dissonance, jugement de goût, sublime, universel

Keywords : aesthetic judgment, beautiful, universal

\section{AUTEUR}

EDOUARD AUJALEU

Professeur agrégé de philosophie, chargé d'enseignement à l'IUFM de Montpellier 